\title{
A Novel Surrogate Safety Indicator Based on Constant Initial Acceleration and Reaction Time Assumption
}

\author{
Adrian Fazekas, Friederike Hennecke, Eszter Kalló, and Markus Oeser \\ Institute of Highway Engineering, RWTH Aachen University, Aachen, Germany \\ Correspondence should be addressed to Adrian Fazekas; fazekas@isac.rwth-aachen.de
}

Received 2 June 2017; Revised 25 October 2017; Accepted 1 November 2017; Published 13 December 2017

Academic Editor: David F. Llorca

Copyright (c) 2017 Adrian Fazekas et al. This is an open access article distributed under the Creative Commons Attribution License, which permits unrestricted use, distribution, and reproduction in any medium, provided the original work is properly cited.

\begin{abstract}
The development of surrogate safety measures has drawn significant research interest in the field of traffic safety analysis. Innovative data sources such as video-based traffic surveillance systems have made it possible to collect large amounts of microscopic traffic data. By deriving traffic safety indicators such as the Deceleration Rate to Avoid a Crash (DRAC) statements concerning traffic safety over a determined road section can be made. This work presents the derivation of a novel surrogate safety indicator based on a Constant Initial Acceleration and reaction time assumption which considers the interaction between vehicles and describes the traffic safety of a road section. The evaluation is based on a video-based microscopic traffic data collection. To examine the efficiency, the new developed indicator is compared to the original Deceleration Rate to Avoid a Crash (DRAC) and the modified indicator (MDRAC) which includes the reaction time. The results showed that the new indicator is more sensitive in detecting critical situations than the other indicators and in addition describes the conflict situations more realistically.
\end{abstract}

\section{Introduction}

As advances in vehicle technologies lead to a continuous reduction of accident counts in traffic, applications of traffic conflict technologies have received increasing attention in the field of traffic safety research. Amundsen [1] defines a traffic conflict as "an observable situation in which two or more road users approach each other in space and time for such an extent that there is a risk of collision if their movement remain unchanged."

A detailed analysis of traffic conflicts can give us a better insight into crash occurrence and thus leads to more efficient traffic safety measures. With the use of conflict indicators, such as the Deceleration Rate to Avoid a Crash, the relevant conflict situations can be identified.

Many of the indicators covered by existing research publications assume an unchanged speed and direction of the conflicting vehicles [2]. This however insufficiently describes the complex reciprocal behavior of the individual vehicles. Furthermore, many of these indicators have boundary conditions on speed. Because of this, car interactions are considered to be safe as long as the following vehicle has a lower speed compared to the leader although the two vehicles might have high speeds and small gaps between each other [3]. Moreover, the reaction time of the road users is often neglected [4].

In this paper, a modified indicator for conflict situations is presented by considering a reasonable reaction time and a more realistic acceleration behavior in the analytical description. The modified indicator is derived and compared with existing indicators based on a data-set of microscopic traffic data collected by computer-vision-based technologies. Furthermore, a discussion of the results is conducted to analyze the benefits of the modified indicator and also further possible developments are presented.

\section{Literature Review}

Critical safety road sections mostly have an inhomogeneous traffic flow characterized by large speed differences leading to an increase in the number and severity of acceleration and deceleration phases [5,6]. A homogenization of the traffic flow can positively increase the safety level on such road sections.

The Deceleration Rate to Avoid a Crash (DRAC) is a suitable method to identify critical road sections, as it covers 
the varying speeds of individual vehicles and derives suitable deceleration phases for the following vehicle [7-9].

DRAC is defined as the minimum required deceleration rate which a vehicle has to apply to avoid a crash with the leading vehicle.

One important factor in the analysis of traffic safety is the minimum time required for the drivers to react to certain situations, also called Perception Reaction Time (PRT).

Kuang et al. [4] argue that the initial determination of DRAC does not consider the PRT of the following vehicle. They modify this specific safety indicator by implementing a factor including PRT. They reach the conclusion that the Modified DRAC (MDRAC) improves crash prediction performance. Wang and Stamatiadis [10-12] have successfully considered the PRT in a number of their studies when evaluating road safety at intersections.

Besides the area of surrogate safety analysis the PRT has been also the focus of other research fields including the planning of roads [13], the associated traffic light systems, and their switching times $[14,15]$.

Even back in 1936, Greenshields [16] conducted a study on the determination of the probability distribution of reaction time. Reaction times are normally approximated with lognormal probability distribution with the parameters $\mu$ and $\sigma^{2}[17$, 18]. In an extended study survey, Green [19] noticed that due to the numerous influencing factors the determination of a uniform reaction time is very difficult.

Another argument often found across publications is the fact that the DRAC is only considering identical movement direction of the conflicting vehicles. Therefore, Wang and Stamatiadis [10] derived the MDRAC for typical crossing and lane-change conflicts.

In order to reach a conclusion about whether a situation is critical or not, the DRAC or the MDRAC has to be compared with a threshold value $[20,21]$. If the identified DRAC or MDRAC is higher than the given threshold the situation is derived to be critical.

\section{Analytical Methods}

DRAC is defined as the minimum required deceleration rate of the following vehicle to avoid a crash with the leading vehicle. The assumption in the original form of the indicator is that the speed of the leading vehicle remains constant. Mathematically, the DRAC is the squared speed difference between the following $V_{2}$ and the leading vehicle $V_{1}$ divided by their (net) distance gap $D_{1-2}[10,22-24]$.

$$
\text { DRAC }= \begin{cases}\frac{\left(V_{2}-V_{1}\right)^{2}}{2 D_{1-2}}, & \text { if } V_{2}>V_{1} \\ 0, & \text { otherwise. }\end{cases}
$$

Kuang et al. [4] add the reaction time of the vehicle following the DRAC obtaining the Modified DRAC (MDRAC). This parameter is calculated as follows:

$$
\text { MDRAC }=\left\{\begin{array}{ll}
\frac{V_{2}-V_{1}}{2(\mathrm{TTC}-R)}, & \text { if TTC }>R \\
\infty, & \text { otherwise. }
\end{array} \quad \forall V_{2}>V_{1}\right.
$$

In (2) $R$ denotes the PRT and TTC represents the time-tocollision value at an initial time $(t=0)$. TTC is defined as the time remaining until a collision between two vehicles occurs [25] assuming a linear trajectory based on the last known speed value:

$$
\mathrm{TTC}= \begin{cases}\frac{D_{1-2}}{V_{2}-V_{1}}, & \text { if } V_{2}>V_{1} \\ \infty, & \text { otherwise }\end{cases}
$$

Besides the TTC, the reaction time $R$ in (2) has a significant influence on the resulting values. Thus, it is sensible to conduct more thorough investigation on this parameter. Green [19] worked on the determination of the reaction time and came to the conclusion that the shortest reaction time is between 0.7 and 0.75 seconds. For the reaction on unexpected but usual signals, such as the activation of the brake lights, approximately 1.25 seconds are necessary. For unexpected events the reaction time is approximately 1.5 seconds. Morita et al. [26] determined throughout their experiments that the reaction time of the following driver lies between 1.3 and 1.6 seconds when the leading vehicle starts breaking and activates the brake lights. Zhang et al. [27] estimate the reaction time on unexpected incidents with visual indications to 1.13 seconds with a standard deviation of 0.52 seconds and on those without visual indications to 1.25 seconds with a standard deviation of 0.60 seconds. For a $90 \%$ percentile the value would be 1.8 or 2.02 seconds. Therefore, two reaction times have been considered for this study. Reaction time values of 1.3 and 2.02 seconds were used for unexpected incidents with and without visual indications, respectively.

As described in the introductory chapters, Mahmud et al. [28] pointed out the fact that the calculation of the DRAC is based on the hypothetical assumption that the speed of the leading vehicle is constant. Furthermore, the trajectory of the second vehicle is also assumed to rely on a constant speed until the time of the reaction. These assumptions or omissions do not adequately cover the complexity of the real driving situation as thorough investigations carried out by Wiedemann [29] have shown that naturalistic driving behavior consists of continuous change of acceleration and speed. This leads to a periodical change in distance between vehicles and also indicates that the driving speed will seldom be constant.

Due to the fact that the DRAC and the MDRAC do not realistically represent the driving behavior by using a constant speed during the time periods in which vehicles are not reacting, the derived values tend to show a lower level of risk. Thus, the only assumption regarding the speed of the vehicles is that they do not change their acceleration or deceleration unless reacting to a new situation. Assumptions on the leading or following vehicle's hypothetical actions are not considered.

By combining the reaction time described above with the more realistic assumption on the acceleration behavior, this work presents a novel indicator for describing risk called Deceleration Rate to Avoid a Crash using Constant Initial Acceleration (DCIA). The detailed derivation of this indicator will be subsequently presented. 


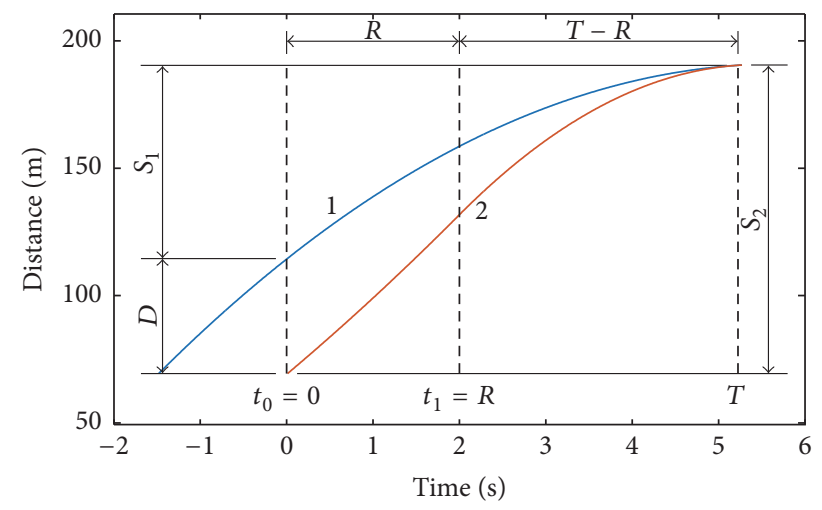

FIgURE 1: Distance-time diagram of the conflict situation. The trajectories of the leading and following vehicle are labeled with the numbers 1 and 2, respectively. The considered time period for the indicator starts at the initial time $t_{0}=0$ with a reaction of the following vehicle at time $t_{1}=R$.

Figure 1 illustrates the distance-time diagram of the considered situation. As shown in the diagram, the following vehicle keeps a constant acceleration until it reacts on the deceleration of the leading vehicle. From this moment the following vehicle will start decelerating with the DCIA value in a way that the net distance between the vehicles at time $T$ is infinitely small and their speeds are equal. This means that the DCIA is the minimal deceleration of the following vehicle so that a collision is avoided.

The mathematical derivation of the DCIA is based on the logic that the distance $S_{2}$ traveled by the following vehicle is equal to the sum of distances $S_{1}$ of the leading one at the moment $T$ with the net distance gap $D$ at time $t_{0}$.

$$
S_{1}(T)+D=S_{2}(T) .
$$

During an arbitrary time $t$ the leading vehicle travels the distance $S_{1}$ :

$$
S_{1}(t)=v_{10} t+\frac{d_{10} t^{2}}{2}
$$

where $v_{10}$ is the speed and $d_{10}$ is the acceleration of vehicle 1 at time $t_{0}$.

The distance, which has been covered by vehicle 2 in the period $t$ after the reaction time $R$, is composed of the distance traveled during the reaction time $R$ and the distance traveled in the time period $t-R$.

$$
S_{2}(t)=v_{20} R+\frac{d_{20} R^{2}}{2}+v_{2}(t-R)+\frac{\operatorname{DCIA}(t-R)^{2}}{2},
$$

where $v_{20}$ is the speed and $d_{20}$ the acceleration at time $t_{0}, v_{2}$ is the speed after the reaction time, and $d_{2}$ is the necessary deceleration rate to prevent a collision or to be able to drive at an infinitely small distance behind the leading vehicle at its speed.

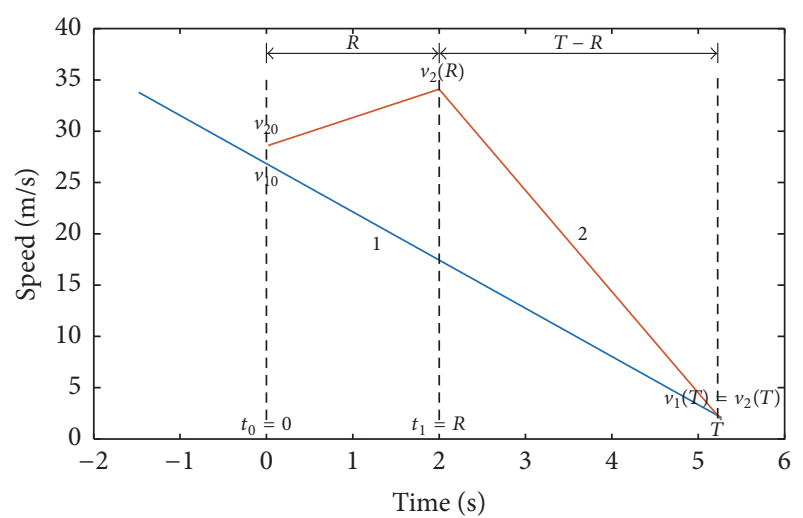

FIGURE 2: Speed-time profile for the conflict situation. The profiles are numbered with 1 and 2 for the leading and following vehicles, respectively, while $v_{10}$ and $v_{20}$ are the corresponding initial speeds.

The DCIA can be determined by substituting (5) and (6) into (4). The required deceleration to avoid an accident is thus calculated according to the following formula:

DCIA

$$
=\frac{2\left(v_{10} T+d_{10} T^{2} / 2+D-v_{20} R-d_{20} R^{2} / 2-v_{2}(T-R)\right)}{(T-R)^{2}} .
$$

In addition to the deceleration rate, the time $T$ is another unknown parameter in this equation. The following contexts are known for the determination of $T$.

The speed of vehicle 1 at an arbitrary time $t$ is

$$
v_{1}(t)=d_{10} t+v_{10}
$$

The speed of vehicle 2 at the time $t_{1}=R$ is

$$
v_{2}(R)=d_{20} R+v_{20} .
$$

The speed of vehicle 2 at the time $t$ after reacting is

$$
v_{2}(t)=d_{2}(t-R)+v_{2}(t) .
$$

According to the definition of the DRAC, the following vehicle should be delayed in such a way that the two vehicles must travel at the same speed at an infinitely small distance one after another, so that $v_{1}(T)=v_{2}(T)$ must apply (see Figure 2).

Therefore,

$$
d_{10} T+v_{10}=\operatorname{DCIA}(T-R)+d_{20} R+v_{20} .
$$

Transformed according to DCIA, the following equation is obtained (see Figure 3):

$$
\text { DCIA }=\frac{d_{10} T+v_{10}-d_{20} R-v_{20}}{T-R} .
$$

$T$ results from (7) and (12):

$$
T=\frac{v_{20} R-v_{10} R-2 D}{v_{10}-d_{10} R-v_{20}+d_{20} R} .
$$




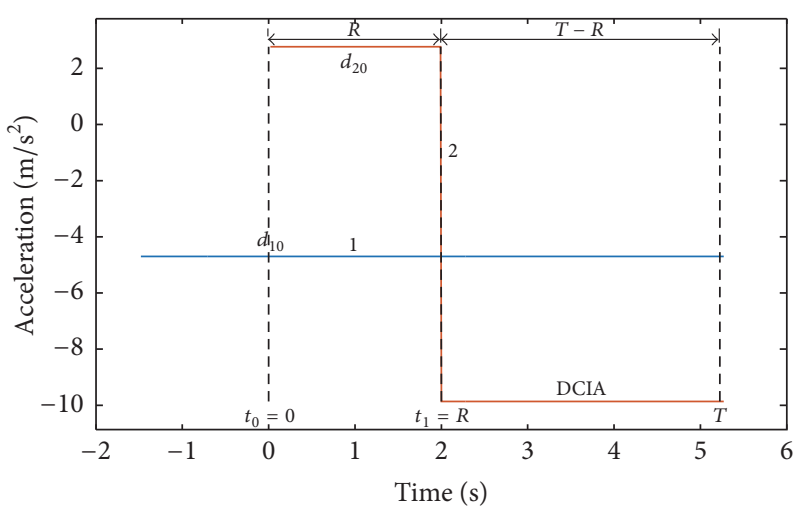

FIGURE 3: Acceleration-time profile for the conflict situation. The profiles are numbered with 1 and 2 for the leading and following vehicles, respectively, while $d_{10}$ and $d_{20}$ are the corresponding initial acceleration.

In contrast to DRAC and MDRAC, DCIA is also suitable for scenarios where the speed of the following vehicle is lower than that of the leading vehicle. Because the DCIA does not assume constant speed profiles but rather a constant acceleration, it is able to identify risky interactions where the following vehicle has a lower speed but a much higher acceleration than the leading vehicle and in combination with a small headway it would come to a crash.

According to AASHTO [20], a traffic situation is considered critical when the DRAC exceeds $3.4 \mathrm{~m} / \mathrm{s}^{2}$. Archer [30] proposes a threshold for critical situation of $3.35 \mathrm{~m} / \mathrm{s}^{2}$. Guido et al. [7] have also chosen a threshold of $3.35 \mathrm{~m} / \mathrm{s}^{2}$ in their study on the traffic safety of a roundabout.

For the evaluation of the DCIA a value less than or equal to 0 means that a collision is prevented without intervention by the following vehicle. A negative value shows the minimum deceleration rate required to avoid a collision by adjusting the speed. In accordance with the studies referenced above a deceleration value greater than $3.4 \mathrm{~m} / \mathrm{s}^{2}$ was chosen as a threshold to indicate a critical situation.

\section{Field Data}

In order to evaluate the methods described above, the conflict indicators are derived from microscopic traffic data. These were extracted from videos recorded within the research project ESIMAS [31] from surveillance cameras in an enclosure tunnel on the German motorway A3, which has 3 lanes. The surveillance cameras are located above the lanes towards Frankfurt also covering an exit lane. The considered section is subject to a speed limit of $100 \mathrm{~km} / \mathrm{h}$.

The video material used for evaluation was recorded from 7:35 a.m. until 7:55 a.m. in summer time with good lighting conditions. It includes traffic jams due to the rush hour traffic.

The derivation of the microscopic traffic data has been performed with software being developed at the Institute of Highway Engineering (RWTH Aachen University) within the research project AUTUKAR regarding automatic video analysis in tunnel surveillance systems (Figure 4). The analysis methods consist of a 3D model based vehicle detection,

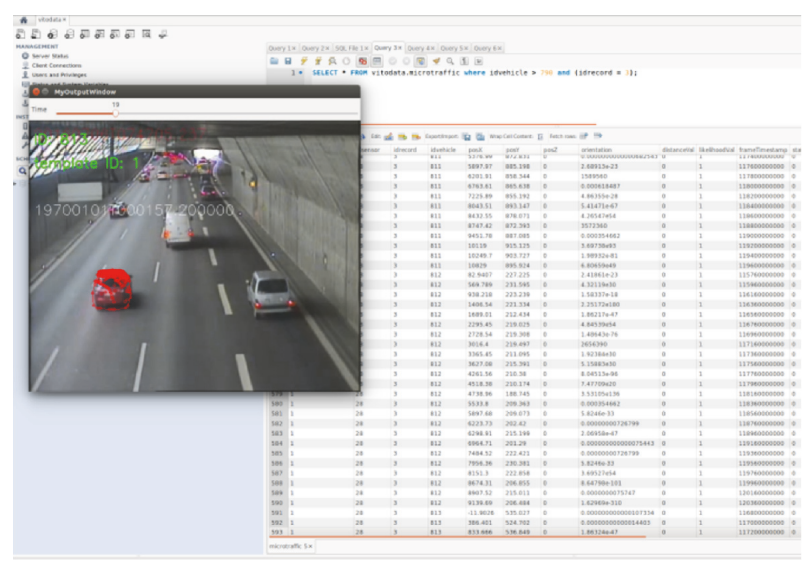

FIgUre 4: Manual position estimation tool GUI. A specific point cloud is moved on the street surface to best fit a vehicle position. The chosen position is then written into a database for further analysis.

classification, and tracking of individual vehicles [32]. The 3D models are point clouds of different vehicle types which are being used to match image features of the real vehicles present in the video. The projection of the point clouds into the two-dimensional image is performed by first assessing the intrinsic and extrinsic parameters of the camera matrix. The assumptions used are that the street is planar and the projection of the vehicle point clouds can only be performed onto this plane. For each vehicle point cloud, an anchor point is computed as the mean point of the outer contour after projecting the point cloud onto the bottom plane. The vehicle positions are determined manually with a graphical user interface by selecting the optimal position of the anchor on the street surface so that the point cloud best matches the image of the vehicle on the street. An automatic analysis is also possible where an image processing based likelihood function finds the position with the best match. While the automatic assessment delivers a higher amount of data, occlusion may lead to false tracking and position estimation. As the main focus of this work is the safety assessment rather than automatic position estimation manual evaluation was used. This gives the best humanly possible accuracy in the position estimation, so that a better analysis of the safety indicator effect can be performed. This manual tagging is also used as proof for the validation of computer vision techniques throughout different research areas.

The accuracy of the data is mostly dependent on the camera calibration itself. An inaccurate camera calibration would lead to inaccurate transformation of pixel coordinates to street surface coordinates and thus to inaccurate distances between vehicles. To determine the calibration matrix, features of the road surface like the lane boundary markings. Based on these markings, virtual positions on the streets are used for calibration as their relative positions can be calculated. The calibration algorithm optimizes the calibration parameters until the back-projection of the input points shows a high accuracy [33]. Thus accuracy of the position estimation is dependent on the accuracy assumed road marking geometry such as the lane width, lengths of 


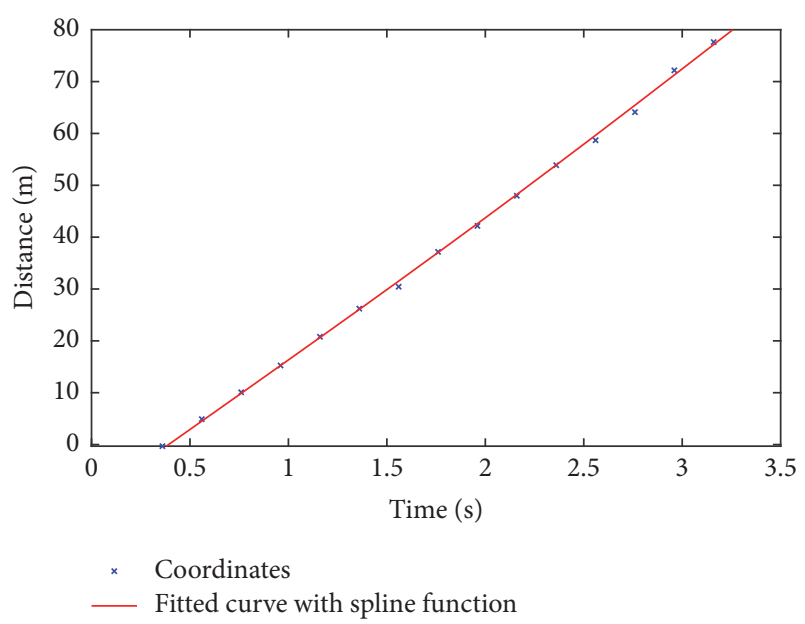

FIGURE 5: A vehicle trajectory with crosses being originally chosen positions and the line being the fitted smoothing spline, which was used for a new oversampling.

the markings, and the gaps between them. Due to the traffic and other safety precautions, another point estimation for $\mathrm{t}$ camera calibration is not possible.

The manual position estimation consists of the first step of choosing the right point cloud which fits best to the real vehicle. After specifying the vehicle type, the position is chosen in different frames, and the two-dimensional coordinates on the street surface with the corresponding timestamp are written into a database. It is important to point out that, with the chosen point cloud, the real length and width of the vehicles are known, which are also used in the calculation of the net distances for the safety indicator assessment.

The coordinates of the vehicles have been imported into MATLAB where the trajectories of the vehicles were estimated by fitting a smoothing spline over the sampled coordinates (Figure 5). When the considered section shows a left curvature, the splines were adjusted, respectively. Using the fitted smoothing splines rather than the original sampled positions, a new sampling time of $0.1 \mathrm{~s}$ could be used for the estimation of the safety indicator. For the estimation of the traffic parameters, such as speed, the values along the smoothing spline are used so that at each $0.1 \mathrm{~s}$ sample the current speed of the vehicle can be estimated.

\section{Results and Discussion}

In order to be able to conduct a thorough validation and discussion of the derived safety indicator, a general macroscopic analysis of the traffic included in the video data needs to be first undertaken.

1,290 vehicles passed through the 80-meter evaluation section in 20 minutes including 115 lane changes. In addition, 1,174 vehicle pairs were identified, which are characterized by the fact that the two consecutive vehicles on the same lane were both present in the 80-meter section at the same time. For the vehicle positions a sampling period of $0.1 \mathrm{~s}$ has been chosen, which has resulted in a total of 16,036 rear-end

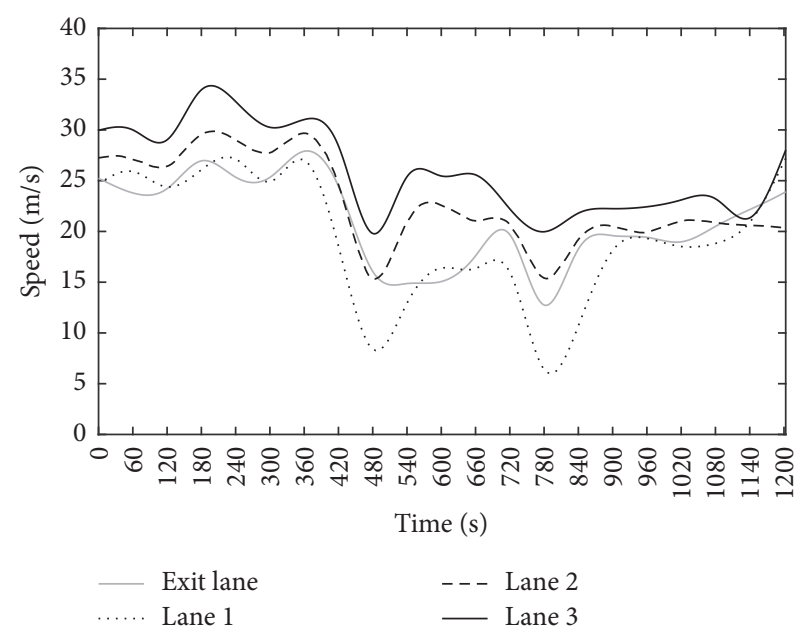

FIGURE 6: Speed-time diagram for the exit lane and the right, middle, and left lane numbered 1,2, and 3, respectively, above the considered time interval.

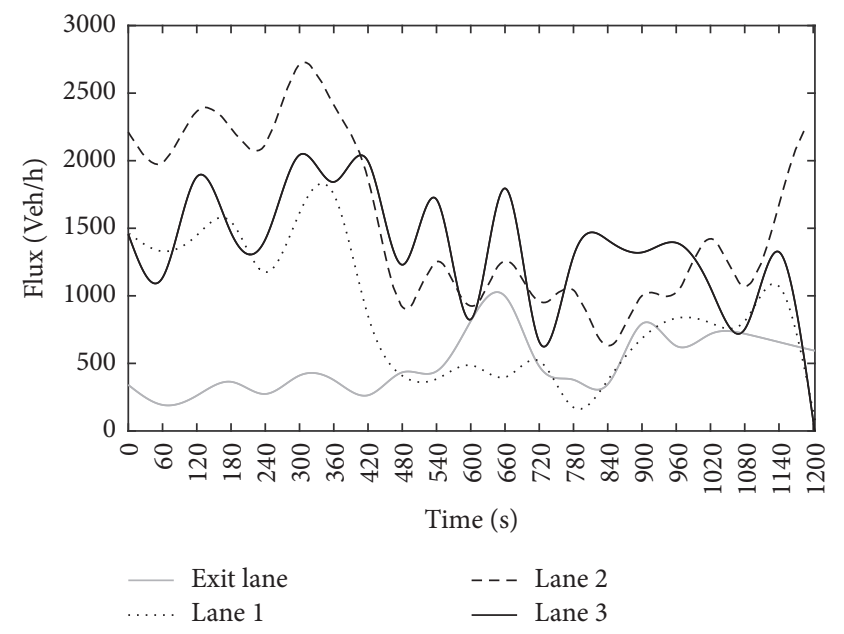

Figure 7: Traffic flux diagram.

scenarios. Out of the entire set of rear-end scenarios 5,842 were recorded on lane 2 .

Figures 6, 7, and 8 show the speed profile, traffic flux, and traffic density for each of the four lanes over the regarded time period, respectively. The values have been determined by running average over 60 previous seconds. In case of speed the values are also a mean over all vehicles present in the considered section.

The traffic is in a free flowing state during the first 300 seconds. The speeds ranged, depending on the considered lane, from approx. 90 to $125 \mathrm{~km} / \mathrm{h}$. Afterwards there was a significant drop in the traffic speed and flux. In the video material it can be seen that the vehicles slowed down due to an event at a further position along the highway section, which resulted in a recognizable change in the speed behavior.

As from second 420 onwards, a traffic backlog was recorded on lane 1 . Starting with second 540, the speed slowly increased and the traffic flow improved. In second 580, a vehicle with a blue flashing light was detected that supports 


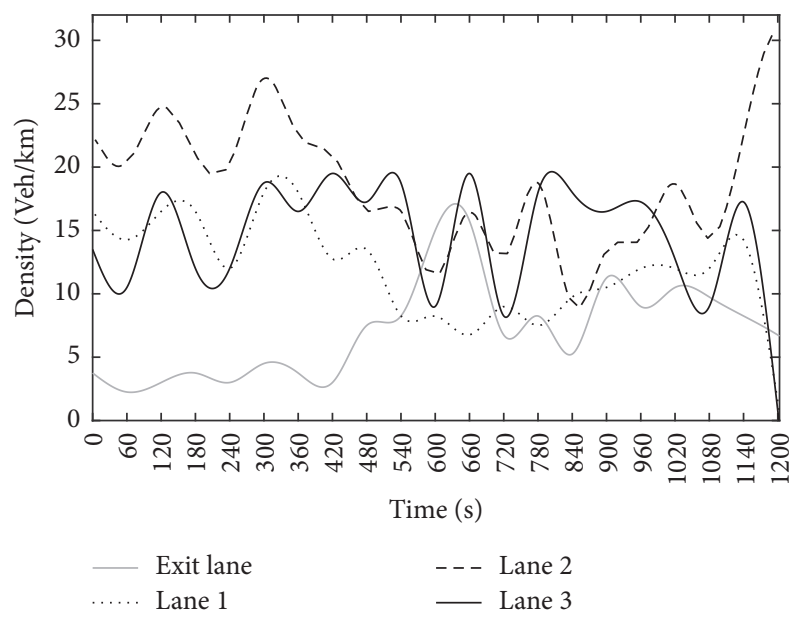

FIgURE 8: Traffic density diagram.

the assumption that an event had occurred in a subsequent section. Based on the traffic parameter graphs of the exit lane it can be seen that after the presence of the blue light many of the vehicles leave the highway. From second 700 onwards, a drop in the speed profile is again observed. From second 750 the traffic density increases in lane 1 and from second 800 onwards it also increases in the other lanes. As from second 840, the speed slowly increases again and the traffic flow improved to a stable traffic state.

With the aim of testing the impact of the PRT and the constant acceleration/deceleration, the performances of traditional and modified surrogate indicators are compared.

The traffic in lane 2 serves as a data base for the derivation of the microscopic traffic safety parameters, since the majority of the vehicles were monitored in this lane. In addition, there is a reduced occlusion compared to lane 1 where high vehicles such as trucks and busses tend to lead to a less accurate position estimation.

In Figures 9-13 the entire height of the bar shows the amount of existing rear-end situations over the defined time intervals. It becomes clear that a higher traffic density leads to a high number of rear-end situations. This is due to the fact that many vehicles are located in this section, and thus many vehicle pairs are registered for assessment. In addition, it can be seen that particularly many rear-end situations occur in the periods of slow moving traffic between seconds 300 to 540 and seconds 780 to 840 . However, the high number of rearend situations does not automatically lead to many critical situations as vehicles tend to drive at low speeds.

Furthermore, the rear-end situations are classified into different criticality groups based on thresholds of the required deceleration rates. The number and proportion of rearend situations in different groups are marked by different color representations inside the bars in the figures. If the determined safety parameter exceeds the threshold value of $3.4 \mathrm{~m} / \mathrm{s}^{2}$ this indicates a critical situation.

As the original DRAC does not exceed the threshold of $3.4 \mathrm{~m} / \mathrm{s}^{2}$ no critical conflicts were identified using this safety indicator (Figure 9). However, it is apparent that starting from

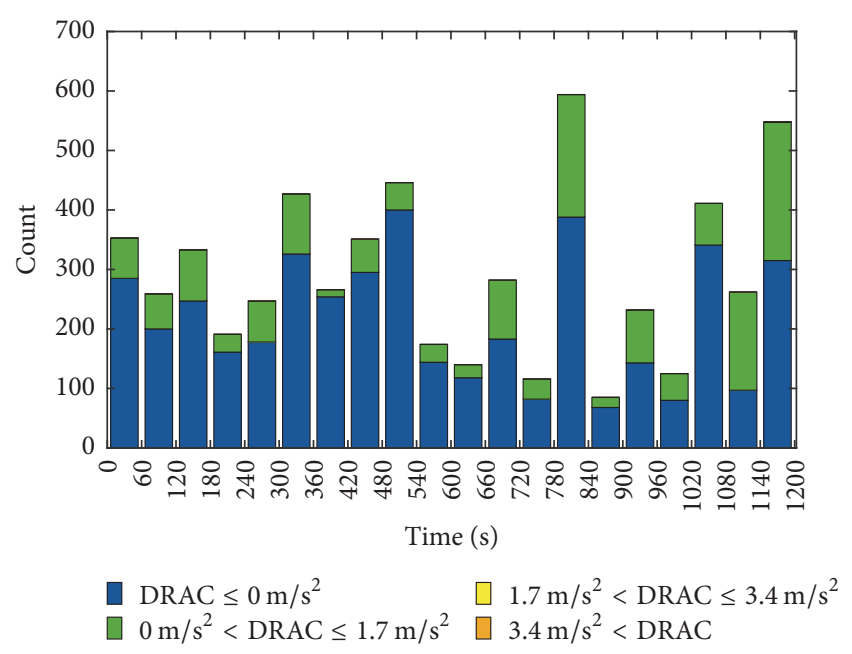

FIGURE 9: DRAC values categorized in four groups over the considered time interval in lane 2.

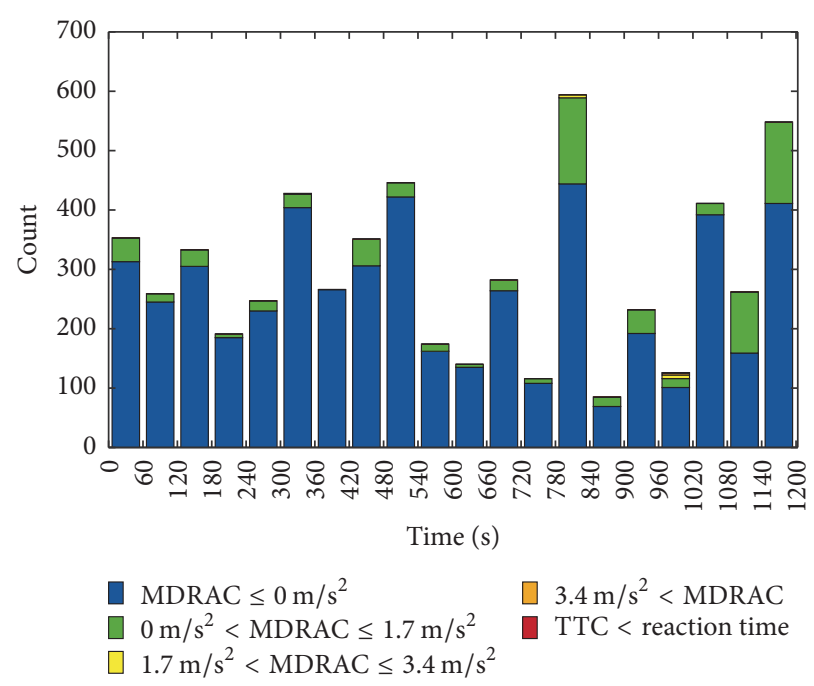

FIGURE 10: MDRAC values calculated with PRT $=1.3 \mathrm{~s}$ categorized in four groups over the considered time interval in lane 2.

second 660 increasingly more rear-end situations occurred where a slight deceleration was necessary to avoid a critical situation.

The modified version of the DRAC, MDRAC, was calculated with two different reaction times, 1.3 seconds and 2.02 seconds. The MDRAC is only calculated when $v_{2}>v_{1}$ and TTC $>R$. However, the number of occurrences where $R>$ TTC can be relevant, as this may indicate an extremely critical situation. This of course does not implicitly mean that an accident occurs but follows from the theoretical assumption of constant speed while the reaction time is measured from the timestamp of the current assessment. For this reason, both criteria are considered.

In Figure 10 the MDRAC was calculated with $R=1.3 \mathrm{~s}$ while in Figure 11 the reaction time value was $R=2.02 \mathrm{~s}$.

It can be seen that values between 1.7 and $3.4 \mathrm{~m} / \mathrm{s}^{2}$ (almost critical situations) can be found at 960 to 1020 seconds 

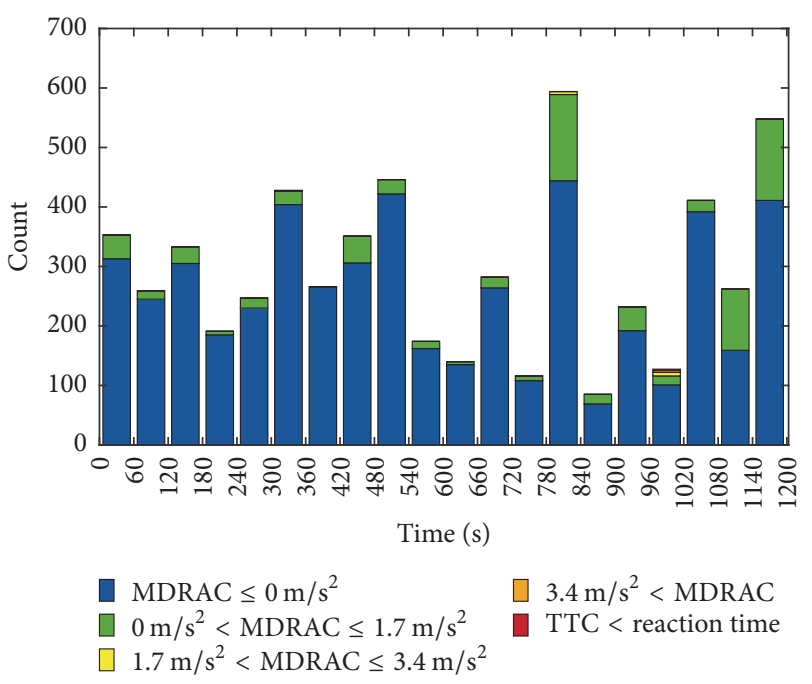

FIGURE 11: MDRAC values calculated with PRT $=2.02 \mathrm{~s}$ categorized in four groups over the considered time interval in lane 2.

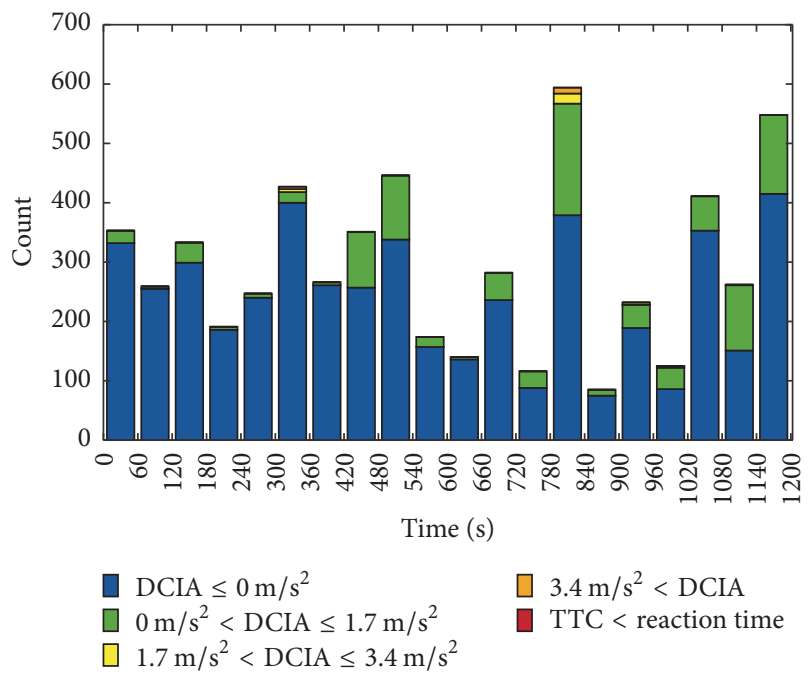

FIGURE 12: DCIA values calculated with PRT $=1.3 \mathrm{~s}$ categorized in four groups over the considered time interval in lane 2.

when the reaction time used is 1.3 seconds (Figure 10). Additionally to this time interval the safety parameter also shows occurrences of values in this criticality group at 780 to 840 seconds when the reaction time is 2.02 seconds (Figure 11). The MDRAC with a reaction time of 2.02 seconds identifies a critical traffic situation at 780-840 seconds.

For further discussions and validation of the DCIA, considering a constant deceleration rather than constant speed has been derived from the data with reaction times of 1.3 seconds and 2.02 seconds (Figures 12 and 13).

Compared to the MDRAC with a reaction time of 1.3 seconds the DCIA results in more critical situations when using the same reaction time. While MDRAC only once identified occurrences of almost critical situations at seconds 960 to 1020 , DCIA registers critical situations at seconds 300 to 360 , seconds 780 to 840 , and second 960 to 1020 , as well as

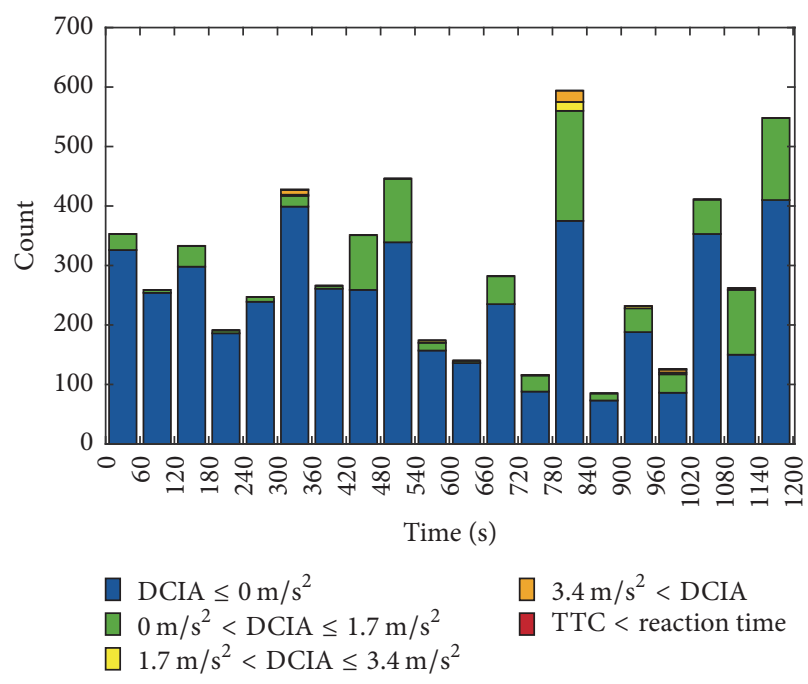

FIGURE 13: DCIA values calculated with PRT $=2.02 \mathrm{~s}$ categorized in four groups over the considered time interval in lane 2 .

almost critical situations at seconds 900 to 960 and seconds 1080 to 1140 .

The phenomenon that the DCIA is more sensitive to critical situations compared to MDRAC is also shown in the comparison between MDRAC and DCIA with a response time of 2.02 seconds. DCIA identifies more critical situations and even 2 situations where the reaction time exceeds the time to collision (TTC).

Overall, it is clear that in the periods of traffic disturbances greater amounts of general rear-end as well as critical rear-end situations occur. On the basis of the critical situations in the period of 960 to 1020 seconds, in which the traffic is almost moving, it can be seen that critical situations or accidents can also occur under normally good circumstances and are attributable to the driving behavior of the vehicle drivers.

The critical situations (deceleration $>3.4 \mathrm{~m} / \mathrm{s}^{2}$ ) were subjected to a detailed analysis. Thereby it was found that four pairs of vehicles are involved in the critical situations, which have differently often led to exceeding the threshold depending on the indicator used (see Table 1). While no critical vehicle combination has occurred using DRAC and MDRAC with $\mathrm{PRT}=1.3 \mathrm{~s}$, there was one occurrence with MDRAC with PRT $=2.02 \mathrm{~s}$, three occurrences with DCIA with $\mathrm{PRT}=1.3 \mathrm{~s}$, and four with DCIA with PRT $=2.02 \mathrm{~s}$.

In addition, it was possible to ascertain through analyzing the video material that two of the four vehicle combinations are lane changes. Although the indicators show that these situations are critical, accelerating before changing the lane for an overtake is a sensible driving behavior which should not be classified as a critical situation.

\section{Conclusion}

In this paper a novel surrogate safety indicator was presented which rather than assuming a constant speed for the conflict prediction only uses a constant acceleration and also includes a theoretical reaction time of the road users. 
TABLE 1: Detailed information about the four identified critical situations.

\begin{tabular}{|c|c|c|c|}
\hline Vehicle-IDs & Time & Safety indicator & Situation \\
\hline $1034 / 1036$ & 964 & $\begin{array}{c}\text { MDRAC, } \mathrm{PRT}=2.0 \mathrm{~s} \\
\text { DCIA, PRT }=1.3 \mathrm{~s} \\
\text { DCIA, PRT }=2.0 \mathrm{~s}\end{array}$ & Lane-change \\
\hline $374 / 375$ & 333 & $\begin{array}{l}\text { DCIA, PRT }=1.3 \mathrm{~s} \\
\text { DCIA, PRT }=2.0 \mathrm{~s}\end{array}$ & Rear-end \\
\hline $872 / 874$ & 798 & $\begin{array}{l}\text { DCIA, PRT }=1.3 \mathrm{~s} \\
\text { DCIA, PRT }=2.0 \mathrm{~s}\end{array}$ & Rear-end \\
\hline $866 / 868$ & 792 & DCIA, $\mathrm{PRT}=2.0 \mathrm{~s}$ & Lane-change \\
\hline
\end{tabular}

The DRAC, MDRAC, and DCIA were assessed using a database of 3D microscopic traffic data gathered from video material by using innovative image processing techniques. A thorough comparison of the three indicators showed that the DCIA is more sensitive in detecting critical situation throughout all criticality groups. The discussion showed that the DCIA is considerably more realistic regarding the description of the dynamic conflict situation.

It was shown that critical situations more often occur at traffic disturbances. Nevertheless, the data showed that critical conflict situations may also occur under circumstances including free flowing traffic due to dangerous driving behavior.

As two of the four identified critical situations detected by the DCIA turned out to be uncritical lane changes, more sophisticated safety indicators need to be developed which can cope with the 3D trajectories of the vehicles. Furthermore, the video-based traffic safety data collection and the subsequent data showed huge potential for safety analysis; thus a higher data volume should be gathered. Further research work should be conducted by comparing large data volumes to real crash data which would lead to a more comprehensive indicator validation.

\section{Conflicts of Interest}

The authors declare that they have no conflicts of interest.

\section{Acknowledgments}

This research is a part of the project "Basic Evaluation for Simulation-Based Crash-Risk-Models: Multi-Scale Modelling Using Dynamic Traffic Flow States" supported by the German Research Directory (Deutsche Forschungsgemeinschaft, DFG). The data-material is based upon work conducted in the project ESIMAS supported by the German Federal Ministry for Economic Affairs and Energy.

\section{References}

[1] F. H. Amundsen, "Proceedings, First Workshop on Traffic Conflicts," in Proceedings of the First Workshop on Traffic Conflicts (Oslo '77), Tekniska Högskolan i Lund, TØI, Oslo, Norway, 1977.
[2] A. R. van der Horst, A Time-Based Analysis of Road User Behaviour in Normal And Critical Encounters, Technische Universität Delft, 1990.

[3] Y. Kuang, X. Qu, and S. Wang, "A tree-structured crash surrogate measure for freeways," Accident, Analysis \& Prevention, vol. 77, pp. 137-148, 2015.

[4] Y. Kuang, X. Qu, J. Weng, and A. Etemad-Shahidi, "How does the driver's perception reaction time affect the performances of crash surrogate measures?" PLoS ONE, vol. 10, no. 9, Article ID e0138617, 2015.

[5] S. Almqvist, C. Hydén, and R. Risser, "Use of speed limiters in cars for increased safety and a better environment," Transportation Research Record: Journal of the Transportation Research Board, no. 1318, pp. 34-39, 1991.

[6] Q. Meng and J. Weng, "Evaluation of rear-end crash risk at work zone using work zone traffic data," Accident, Analysis \& Prevention, vol. 43, no. 4, pp. 1291-1300, 2011.

[7] G. Guido, F. F. Saccomanno, A. Vitale, V. Astarita, and D. C. Festa, "Comparing safety performance measures obtained from video capture data," Journal of Transportation Engineering, vol. 137, no. 7, pp. 481-492, 2011.

[8] V. Astarita, G. Guido, A. Vitale, and V. P. Giofré, "A new microsimulation model for the evaluation of traffic safety performances," European Transport/Trasporti Europei, no. 51, pp. 1-16, 2012.

[9] D. Gettman and L. Head, "Surrogate safety measures from traffic simulation models," Transportation Research Record, no. 1840, pp. 104-115, 2003.

[10] C. Wang and N. Stamatiadis, "Surrogate safety measure for simulation-based conflict study," Transportation Research Record, no. 2386, pp. 72-80, 2013.

[11] C. Wang and N. Stamatiadis, "Derivation of a new surrogate measure of crash severity," Transportation Research Record, vol. 2432, pp. 37-45, 2014.

[12] C. Wang and N. Stamatiadis, "Evaluation of a simulation-based surrogate safety metric," Accident, Analysis \& Prevention, vol. 71, pp. 82-92, 2014.

[13] D. B. Fambro, K. Fitzpatrick, and R. J. Koppa, "New stopping sight distance model for use in highway geometric design," Transportation Research Record, no. 1701, pp. 1-8, 2000.

[14] H. Rakha, I. El-Shawarby, and J. R. Setti, "Characterizing driver behavior on signalized intersection approaches at the onset of a yellow-phase trigger," IEEE Transactions on Intelligent Transportation Systems, vol. 8, no. 4, pp. 630-640, 2007. 
[15] F. Wang, K. Tang, and K. Li, "A stochastic computational model for yellow time determination and its application," Journal of Advanced Transportation, vol. 49, no. 3, pp. 457-474, 2015.

[16] B. D. Greenshields, "Reaction time in automobile driving," Journal of Applied Psychology, vol. 20, no. 3, pp. 353-359, 1936.

[17] G. Taoka, "Break reaction times of unalerted drivers," ITE Journal, vol. 59, no. 3, pp. 19-21, 1989.

[18] H. Summala, "Brake reaction times and driver behavior analysis," Transportation Human Factors, vol. 2, no. 3, pp. 217-226, 2000.

[19] M. Green, "“How long does it take to stop?" methodological analysis of driver perception-brake times," Transportation Human Factors, vol. 2, no. 3, pp. 195-216, 2000.

[20] American Association of State Highway and Transportation Officials, "A Policy on Geometric Design of Highways and Streets," AASHTO, 2004.

[21] J. Archer, Methods for the Assessment and Prediction of Traffic Safety at Urban Intersections and their Application in Microsimulation Modelling, Academic Thesis, Royal Institute of Technology Stockholm, 2004.

[22] A. R. Mamdoohi, M. F. Zavareh, C. Hydén, and T. Nordfiærn, "Comparative analysis of safety performance indicators based on inductive loop detector data," PROMET-Traffic \& Transportation, vol. 26, no. 2, pp. 139-149, 2014.

[23] L. T. Truong, M. Sarvi, G. Currie, and T. M. Garoni, "How many simulation runs are required to achieve statistically confident results: a case study of simulation-based surrogate safety measures," in Proceedings of the 18th IEEE International Conference on Intelligent Transportation Systems (ITSC '15), pp. 274-278, Gran Canaria, Spain, September 2015.

[24] G. Guido, V. Astarita, V. Giofré, and A. Vitale, "Safety performance measures: a comparison between microsimulation and observational data," in Proceedings of the 14th Meeting of the Euro Working Group on Transp. - In Quest for Adv. Models, Tools, and Methods for Transp. and Logist., EWGT, 26th Mini-EURO Conf. - Intelligent Decis. Making in Transp. and Logist., MEC, 1st Eur. Sci. Conf. on Air Transp. - RH, vol. 20, pp. 217-225, September 2011.

[25] Y. Kuang and X. Qu, "A Review of Crash Surrogate Events," in Proceedings of the 2nd International Conference on Vulnerability and Risk Analysis and Management, ICVRAM 2014 and the 6th International Symposium on Uncertainty Modeling and Analysis (ISUMA '14), pp. 2254-2264, July 2014.

[26] K. Morita, M. Sekine, and T. Okada, "Factors with the greatest influence on drivers' judgment of when to apply brakes," in Proceedings of the 2006 SICE-ICASE International Joint Conference, pp. 5044-5049, Busan, Korea, October 2006.

[27] Y. Zhang, E. K. Antonsson, and K. Grote, "A new threat assessment measure for collision avoidance systems," in Proceedings of the IEEE Intelligent Transportation Systems Conference (ITSC '06), pp. 968-975, September 2006.

[28] S. M. S. Mahmud, L. Ferreira, M. S. Hoque, and A. Tavassoli, "Application of proximal surrogate indicators for safety evaluation: a review of recent developments and research needs," IATSS Research, 2016.

[29] R. Wiedemann, Simulation des Straßenverkehrsflusses, Universität Karlsruhe, 1974.

[30] J. Archer, Indicators for Traffic Safety Assessment And Prediction And Their Application in Micro-Simulation Modelling: A Study of Urban And Suburban Intersections, Royal Institute of Technology Stockholm, 2005.
[31] Bundesanstalt für Straßenwesen, "Echtzeit-SicherheitsManagement-System für Straßentunnel (ESIMAS)," http:// www.esimas.de/.

[32] A. Fazekas, M. Bommes, and M. Oeser, "Vehicle Tracking using 3D Particle Filter in Tunnel Surveillance and Incident Detection," in Proceedings of the 3rd International Conference on : Models and Technologies for Intelligent Transportation Systems 2013, vol. no. 3, pp. 213-222, 2013.

[33] Z. Zhang, "A flexible new technique for camera calibration," IEEE Transactions on Pattern Analysis and Machine Intelligence, vol. 22, no. 11, pp. 1330-1334, 2000. 


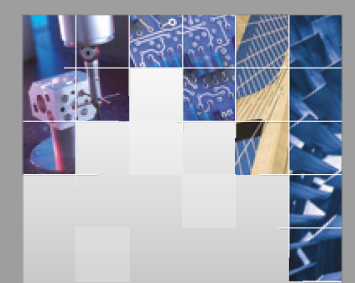

\section{Enfincering}
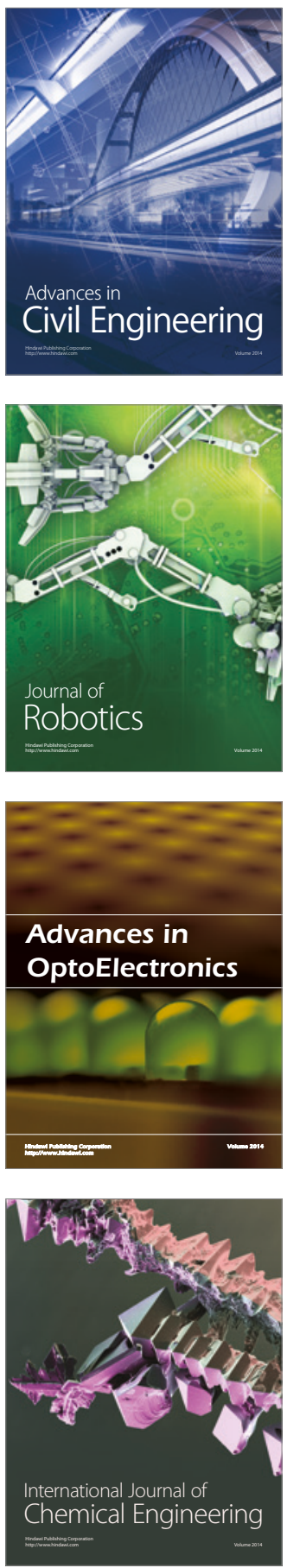

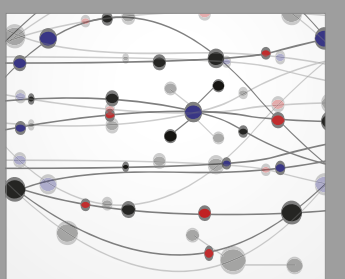

The Scientific World Journal

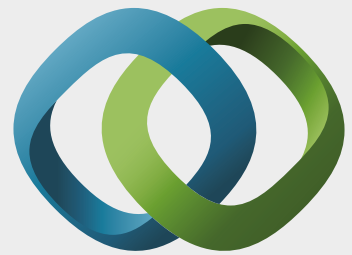

\section{Hindawi}

Submit your manuscripts at

https://www.hindawi.com
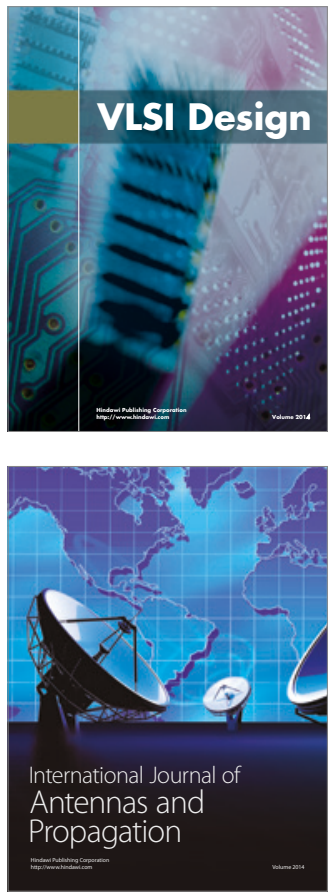

\section{Rotating}

Machinery
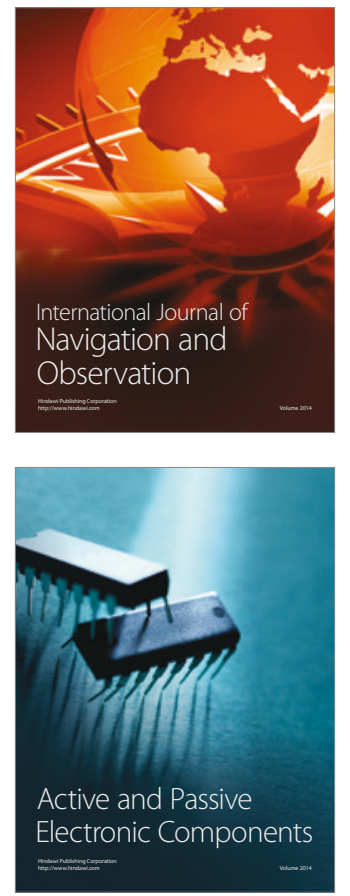
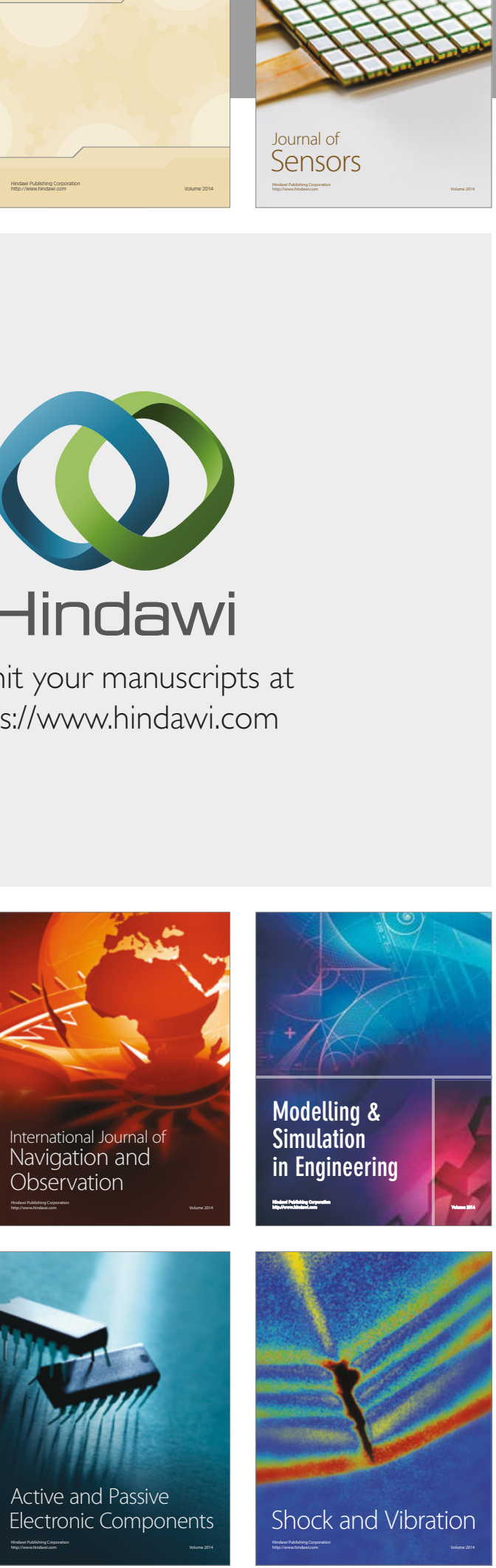
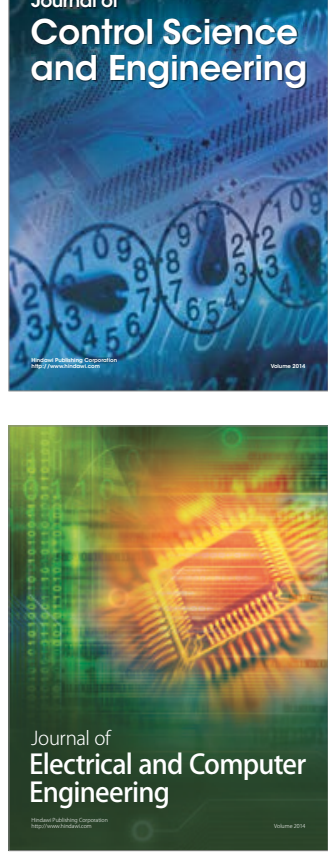

Distributed

Journal of

Control Science

and Engineering
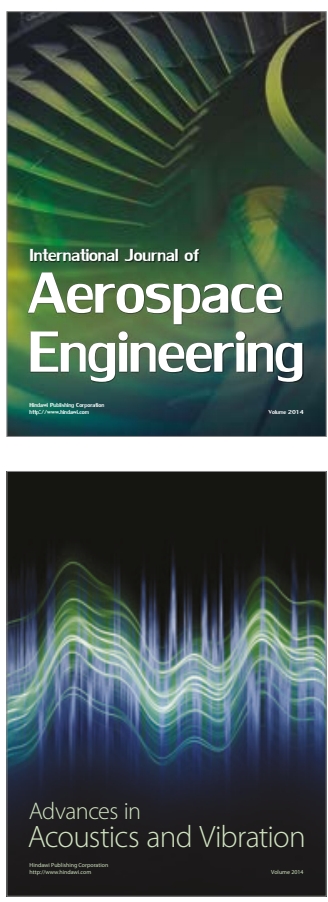

Sensor Networks 